\title{
Deep vein thrombosis in COVID-19 patients in general wards: prevalence and association with clinical and laboratory variables
}

\author{
Anna Maria lerardi ${ }^{1}\left[\right.$ - Nicola Gaibazzi ${ }^{2} \cdot$ Domenico Tuttolomondo $^{2} \cdot$ Stefano Fusco $^{3} \cdot$ Vincenzo La Mura $^{4}$. \\ Flora Peyvandi ${ }^{4}$. Stefano Aliberti ${ }^{5,6} \cdot$ Francesco Blasi $^{5,6} \cdot$ Diletta Cozzi $^{7} \cdot$ Gianpaolo Carrafiello $^{2,8}$. \\ Massimo De Filippo ${ }^{9}$
}

Received: 24 August 2020 / Accepted: 15 November 2020 / Published online: 19 January 2021

(C) Italian Society of Medical Radiology 2021

\begin{abstract}
Background Preliminary reports suggest a hypercoagulable state in COVID-19. Deep vein thrombosis (DVT) is perceived as a frequent finding in hospitalized COVID-19 patients, but data describing the prevalence of DVT are lacking.

Objectives We aimed to report the prevalence of DVT in COVID-19 patients in general wards, blinded to symptoms/signs of disease, using lower extremities duplex ultrasound (LEDUS) in random patients. We tested the association of DVT with clinical, laboratory and inflammatory markers and also reported on the secondary endpoint of in-hospital mortality.

Patients/Methods $n=263$ COVID-19 patients were screened with LEDUS between March 01, 2020 and April 05, 2020 out of the overall $n=1012$ admitted with COVID-19.

Results DVT was detected in $n=67$ screened patients (25.5\%), $n=41$ patients $(15.6 \%)$ died during the index hospitalization. Multiple logistic regression demonstrated that only C-reactive protein (odds ratio 1.009, 95\% CI 1.004-1.013, $p<0.001$ ) was independently associated with the presence of DVT at LEDUS. Both age (odds ratio 1.101, 95\% CI 1.054-1.150, $p<$ 0.001 ) and C-reactive protein (odds ratio 1.012, 95\% CI 1.006-1.018, $p<0.001$ ) were instead significantly independently associated with in-hospital mortality.

Conclusions The main study finding is that DVT prevalence in COVID-19 patients admitted to general wards is $25.5 \%$, suggesting it may be reasonable to screen COVID-19 patients for this potentially severe but treatable complication, and that inflammation, measured with serum C-reactive protein, is the main variable associated with the presence of DVT, where all other clinical or laboratory variables, age or D-dimer included, are instead not independently associated with DVT.
\end{abstract}

Keywords Deep vein thrombosis $\cdot$ Screening $\cdot$ COVID-19 $\cdot$ Duplex ultrasound $\cdot$ C-reactive protein

Anna Maria Ierardi

amierardi@yahoo.it

1 Radiology Department, Fondazione IRCCS Cà Granda Ospedale Maggiore Policlinico, Milan, Italy

2 Cardiology Department, Azienda Ospedaliero-Universitaria di Parma, Parma, Italy

3 School of Radiology, University of Milan, Milan, Italy

4 Fondazione IRCCS Ca' Granda, Ospedale Maggiore Policlinico, U.O.C. Medicina Generale Emostasi e Trombosi, University of Milan, Milan, Italy
5 UOC Pneumologia, Fondazione IRCCS Ca' Granda Ospedale Maggiore Policlinico, Milan, Italy

6 Department of Pathophysiology and Transplantation, Università degli Studi di Milano, Milan, Italy

7 Department of Radiology, Azienda Ospedaliero-Universitaria Careggi, Florence, Italy

8 Department of Health Sciences, Università degli Studi di Milano, Milan, Italy

9 Department of Medicine and Surgery (DiMeC), Unit of Radiology, University of Parma, Parma, Italy 


\section{Introduction}

Acute respiratory disease from SARS-CoV-2 (COVID-19) or coronavirus disease 2019, is an infectious, mainly respiratory disease caused by a virus named SARS-CoV-2 and it is the cause of the ongoing worldwide pandemic in 2020.

One of the reasons that explains the rapid and uncontrolled spread of the virus is due to the relatively long incubation period, in which the infected subjects typically show no, mild or non-respiratory symptoms and whose duration can vary between 2 and 14 days during which the host can be contagious. Inter-human transmission occurs by contact with infected secretions or by air (coughing or sneezing) droplets [1, 2].

A key step in the diagnostic process of patients suspected for COVID-19 is the use of chest computed tomography. The HRCT (high-resolution computed tomography) without the use of contrast rapidly allows the radiographic diagnosis of interstitial pneumonia and provide helpful prognostic information [3-5]. Chest X-ray has also proved useful in the emergency setting as a quantitative method of the extent of SARS-CoV-2 pneumonia, correlating with an increased risk of intensive care unit admission [6].

There are several reasons why a patient affected by COVID-19 may be predisposed to concomitant thrombotic and thromboembolic disease.

Preliminary reports suggest the presence of a hypercoagulable state in COVID-19, with several (and partly conflicting) haemostatic abnormalities being documented, such as high D-dimer and other fibrin degradation products, high fibrinogen, prolonged activated partial thromboplastin time, positivity for lupus anticoagulant and other abnormalities, suggesting some forms of undetermined coagulopathy [7-9]. It is unknown whether these haemostatic changes are directly caused by SARS-CoV-2 or rather a consequence of the cytokine storm following the systemic inflammatory response syndrome, as observed in other viral disease [10-13].

In this context, deep vein thrombosis (DVT) is perceived by clinicians on the field as a frequent finding in hospitalized COVID-19 patients, although data describing the true prevalence of DVT in COVID-19 are completely lacking. The few existing published reports on DVT in hospitalized patients with COVID-19 are either specifically collected only in intensive care units or they describe overall thromboembolic events. Existing reports either enrolled patients based on clinical signs or symptoms of DVT or of thromboembolic events in general, performing duplex ultrasound because of this very high index of clinical suspicion, or addressed only patients admitted to intensive care units [14-18]; by so doing such studies select highest-risk, symptomatic patients, in particular excluding the ones with mild or no symptoms or signs of DVT, finally reporting biased data on DVT prevalence.

We aim to (a) describe the prevalence of DVT by screening COVID-19 patients independently from their symptomatic status for DVT, using lower extremities duplex ultrasound (LEDUS) scan in random patients admitted to general wards (low or mid intensity care units), where the vast majority of COVID-19 patients are initially admitted, (b) test the potential association of DVT with clinical, laboratory and inflammatory markers, (c) report on the secondary end point of in-hospital mortality.

\section{Methods}

This cross-sectional, single-centre study used a complete radiologist-performed LEDUS scan to assess the prevalence of DVT of the lower extremities in laboratory-confirmed COVID-19 patients between March 01, 2020 and April 05, 2020. In-hospital mortality was also collected as a secondary end point.

\section{Patients selection}

We screened with LEDUS a random sample of patients with laboratory-confirmed COVID-19, admitted and treated in non-intensive care units (low-intensity and mid-intensity care units) dedicated to COVID-19; such patients were initially admitted because of clinically-suspected COVID-19, but only the ones in whom the diagnosis was then confirmed by SARS-CoV-2 viral nucleic acid assay in nasopharyngeal swabs were selected for screening. We did not include patients who were diagnosed with COVID-19 during hospital stay, but were admitted for other medical conditions.

\section{Index screening test}

Patients were selected for LEDUS screening in a quasi-random fashion. In fact LEDUS random screening was clinically felt appropriate ad spontaneously implemented by the radiology department, after the notion spread that COVID19 could be a systemic pro-thrombotic disease. Based on the voluntary availability of one of the radiologists in charge on each day of the week, he/she was randomly assigned one of the designated COVID-19 units of the Fondazione IRCCS Cà Granda Ospedale Maggiore Policlinico, rotating on a daily basis, to perform bedside LEDUS in a minimum of 10 random patients for each working day. Patients to be scanned were chosen starting alternatively from the first or last room in the ward depending on the day. 


\section{Clinical variables}

The demographics, traditional cardiovascular risk factors and the available laboratory blood tests (C-reactive protein, D-dimer and Fibrinogen) were the independent variables to be tested for an association with the primary endpoint (DVT) or with the secondary endpoint (in-hospital death).

\section{Endpoints}

The presence of DVT at the full LEDUS scan was the primary endpoint; in-hospital death, collected at least 30 days after admission was the secondary endpoint.

This study complied with the edicts of the 1975 Declaration of Helsinki and was approved by our Institutional Review Board.

\section{Statistical methods}

No statistical sample size calculation was performed a priori, and sample size was equal to the number of patients enrolled during the study period. Categorical variables are expressed as number of patients (percentage) with $95 \%$ CIs, and continuous variables as mean (SD) or median (interquartile range [IQR]) as appropriate. The means for continuous variables were compared using independent group $t$ tests when the data were normally distributed, otherwise, the MannWhitney test was used. Proportions for categorical variables were compared using the $\chi^{2}$ test, although the Fisher exact test was used when data were limited. Stepwise multiple logistic regression was used to assess the relationship between, first the demographic and clinical variables and then adding laboratory variables, and the end point of the detection of DVT at LEDUS; secondarily we tested the end point of in-hospital mortality. All variables with $p<0.1$ on univariable analysis were considered for the inclusion into multivariable logistic regression models. A 2 -sided $p<0.05$ was considered statistically significant. All statistical analyses were performed with Stata statistical software, version 15.0 (StataCorp LLC, USA).

\section{Results}

Two hundred and sixty three patients laboratory-confirmed COVID-19 patients were randomly screened with LEDUS, out of the overall 1012 admitted to the hospital with confirmed COVID-19 in the same period of time. Mean age was $63 \pm 15, n=175$ patients were male $(66.5 \%)$ and $n=41$ patients $(15.6 \%)$ died during their index hospitalization, $n=222(84.4 \%)$ were discharged home alive. DVT was detected in 67 of the 263 screened patients (25.5\%), among which 22 DVT were bilateral (32.8\%). In 21 patients DVT was found in the femoral veins $(31.3 \%), 18$ in the popliteal veins (26.9\%) and 28 in the calf veins (41.8\%) (Table 1 reports baseline characteristics and frequencies of
Table 1. Demographics, clinical, laboratory tests, LEDUS results and frequency of end points.

\begin{tabular}{ll}
\hline No.(\%, if not otherwise specified) & Total \\
\hline Demographics & \\
Number of patients & 263 \\
Age, median [lower-upper quartile], y & $63[54-76]$ \\
Female sex & $88(33)$ \\
Risk factors and patient history & \\
Hypertension & $128(49)$ \\
Dyslipidaemia & $27(10)$ \\
Current Smoker & $17(6)$ \\
Diabetes mellitus & $53(20)$ \\
Obesity & $45(17)$ \\
History of prior DVT & $7(3)$ \\
Laboratory blood tests & \\
C-reactive protein, median [lower-upper quartile] (mg/l) & $52[13-115]$ \\
D-dimer, median [lower-upper quartile] ng/ml & $1332[809-3779]$ \\
Fibrinogen, [lower-upper quartile] ng/dl & $536[390-691]$ \\
End points & \\
Patients with DVT at LEDUS & $67(25.5)$ \\
Bilateral DVT & $22(33)$ \\
Femoral veins DVT & $21(31)$ \\
Patients who died in-hospital & $41(16)$ \\
\hline
\end{tabular}

$D V T$ deep vein thrombosis, LEDUS lower extremities duplex ultrasound 
end points). LEDUS was performed at a mean $9 \pm 6$ day after hospital admission. All patients, from admission and at least until the day LEDUS was performed, were per hospital protocol treated with prophylactic doses of weight-adjusted enoxaparin (100 international units per kilogram, once per day, the dose being halved in severe chronic kidney disease).

\section{Primary end point}

Figure 1 shows the box and whisker plots graphically demonstrating the distribution of continuous independent variables-age, C-reactive protein, D-dimer and fibrinogen - in patients with and without the finding of DVT at LEDUS.

When testing univariable association of the demographic or clinical variables (Table 2, left column) only the presence of hypertension (odds ratio 1.858, 95\% CI 1.021-3.380, $p=0.042$ ) was significantly associated with DVT; among laboratory variables, C-reactive protein (odds ratio 1.009, 95\% CI 1.005-1.012, $p<0.001$ ), D-dimer (odds ratio $1.000,95 \%$ CI $1.000-1.000, p=0.021)$ and fibrinogen (odds ratio $1.003,95 \%$ CI 1.002-1.004, $p<0.001$ ) were also significantly associated with DVT. Since C-reactive protein and fibrinogen were strongly and significantly correlated $(r=0.610, p<0.001)$ only $\mathrm{C}$-reactive protein and not fibrinogen (mainly because $\mathrm{C}$-reactive protein use is more widespread) were inserted in the multiple logistic regression analysis. Stepwise multiple logistic regression demonstrated that only C-reactive protein (odds ratio 1.009, $95 \%$ CI 1.004-1.013, $p<0.001$ ) was finally independently associated with the presence of DVT at LEDUS (Table 2, right columns).

\section{Secondary end point}

Regarding the secondary end point of in-hospital mortality, age (odds ratio $1.089,95 \%$ CI 1.056-1.12, $p<0.001$ ), dyslipidaemia (odds ratio 3.286, 95\% CI 1.216-8.877, $p=0.019$ ) and C-reactive protein (odds ratio $1.006,95 \% \mathrm{CI}$ $1.005-1.013, p<0.001$ ) were significantly associated in univariable assessment, while in multivariable stepwise logistic

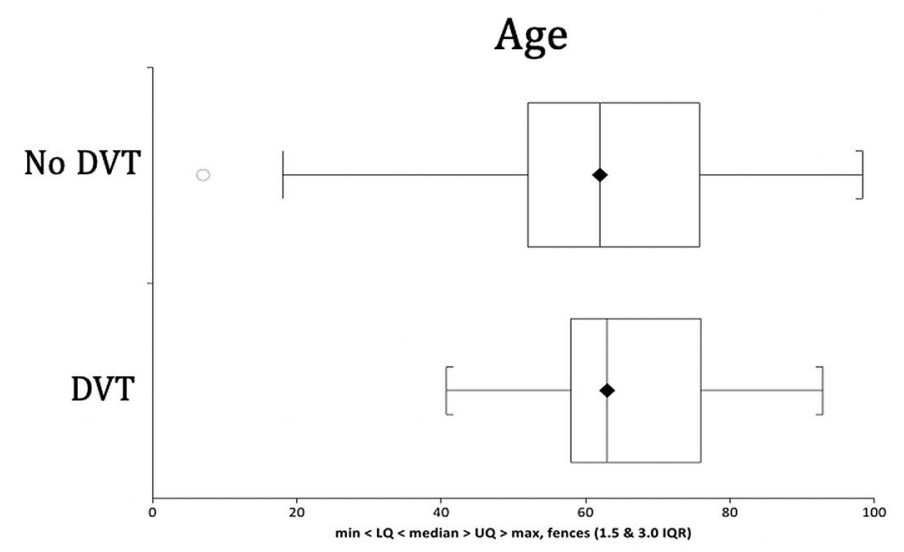

C-reactive protein

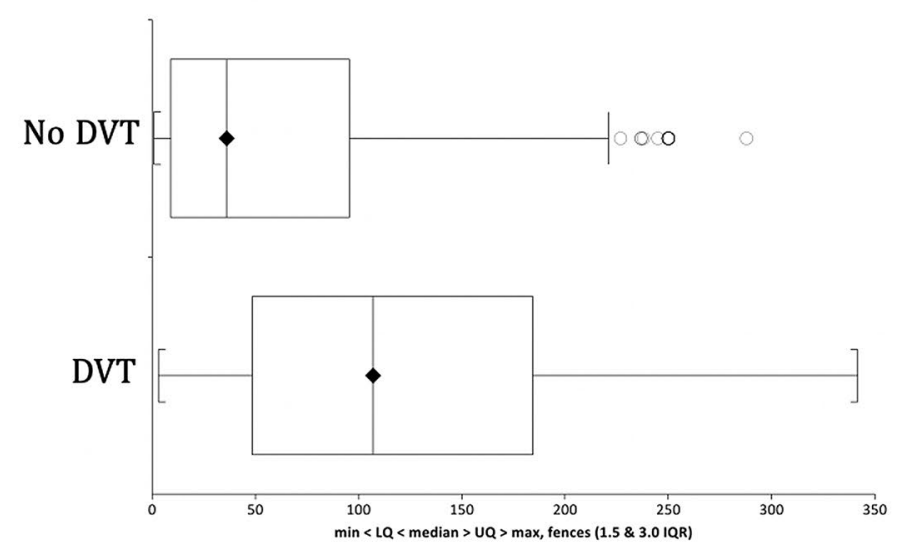

\section{D-dimer}

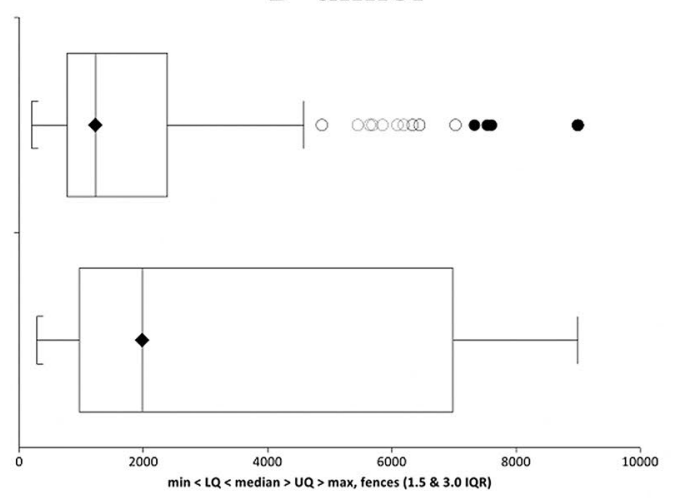

Fibrinogen

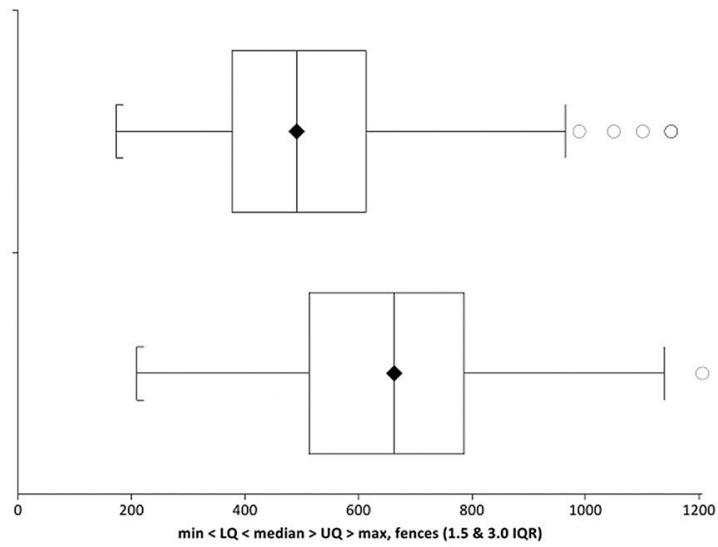

Fig. 1 Distribution of main continuous independent variables in the groups with and without deep vein thrombosis (DVT) at lower extremities duplex ultrasound. DVT: deep vein thrombosis 


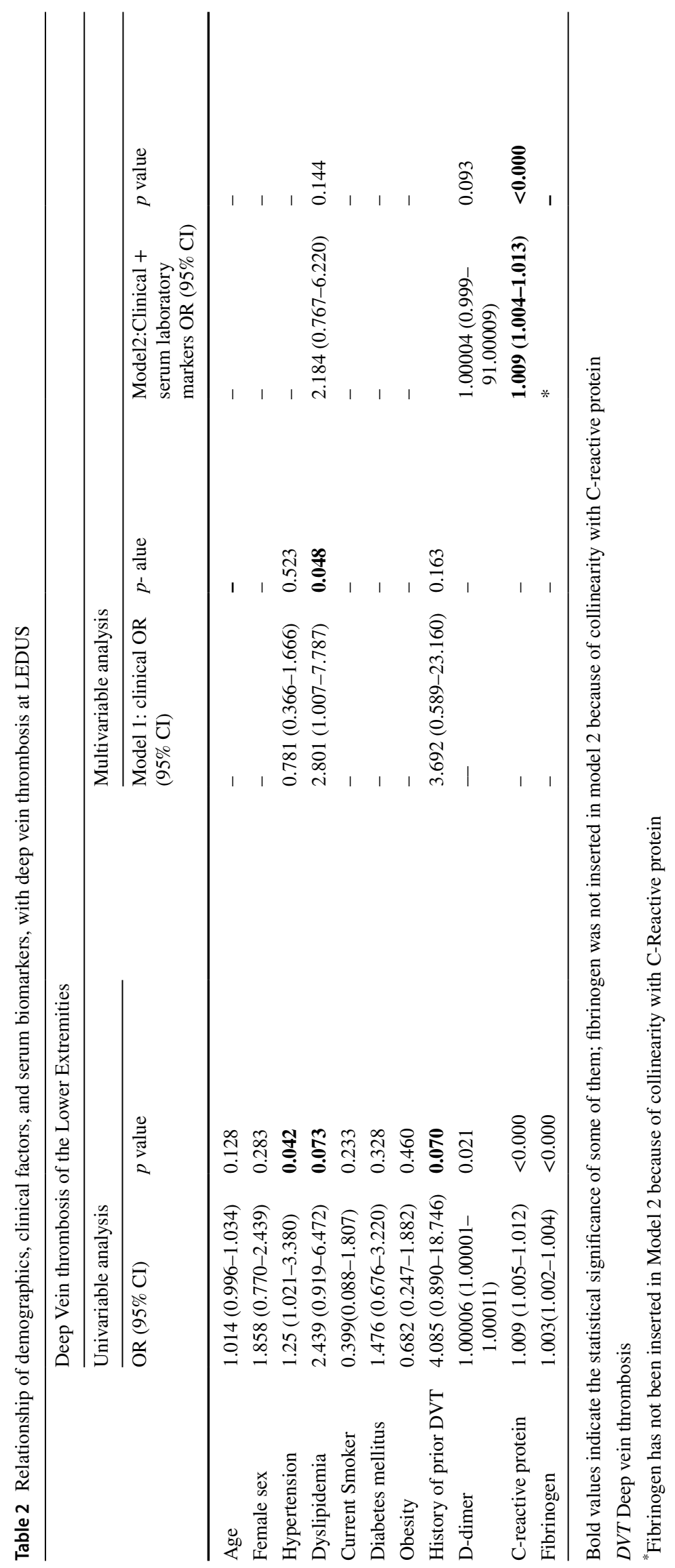


regression, only age (odds ratio $1.101,95 \%$ CI $1.054-1.150$, $p<0.001$ ) and C-reactive protein (odds ratio $1.012,95 \% \mathrm{CI}$ $1.006-1.018, p<0.001)$ were significantly and independently associated with in-hospital mortality.

\section{Discussion}

The main finding of this screening study is the description that DVT in random, laboratory-confirmed COVID-19 patients admitted to general wards is $25.5 \%$. The prevalence of DVT in patients admitted because of COVID-19 is here reported for the first time, to the best of our knowledge, using a LEDUS screening strategy in a random sample of subjects with COVID-19. We did not assess patients preselected based either on their extreme clinical severity (as done when assessing only patients in intensive care units) or based on the presence of symptoms and signs of DVT. We think the current study supports the concept that approximately one quarter of COVID-19 patients admitted to general wards in fact have DVT, by so doing suggesting it may be reasonable to screen COVID-19 patients for this potentially severe but treatable complication.

The second finding is that the grade of inflammation, in this case measured with serum C-reactive protein, is the main (and only independent) variable associated with the presence of DVT, where all other clinical or laboratory variables, age or D-dimer included, are instead not independently associated with DVT.

Interestingly, the commonly used threshold of a D-dimer $>500 \mathrm{ng} / \mathrm{ml}$ to suspect DVT (or other thromboembolic events) in general patients did not fit in the specific COVID-19 setting, in which D-dimer is in fact known to be generally increased [19]. For example, in the current study the median D-dimer value was $1332 \mathrm{ng} / \mathrm{ml}$ (lowerupper quartile $809-3779 \mathrm{ng} / \mathrm{ml}$ ) and the use of the $<500$ $\mathrm{ng} / \mathrm{ml}$ cut-off would classify only 28 patients $(11 \%)$ as lowrisk for DVT; unfortunately, this $500 \mathrm{ng} / \mathrm{ml} \mathrm{D}$-dimer cutoff also demonstrated suboptimal sensitivity in our cohort, "missing" the DVT diagnosis in 3 patients out of the 28 with a D-dimer value lower than $500 \mathrm{ng} / \mathrm{ml}$. The 3 patients with DVT and D-dimer $<500 \mathrm{ng} / \mathrm{ml}$, however, had very high C-reactive protein values, which highlights the role of inflammation. In fact, if we alternatively approached COVID-19 patients starting from C-reactive protein values, it is of interest that in the 36 patients (14\%) with normal C-reactive protein at admission (normal range is $0.5-5$ $\mathrm{mg} / \mathrm{l}$ ), no one had DVT at LEDUS, in spite of a frequently high D-dimer ( $>500 \mathrm{ng} / \mathrm{ml}$ in 26 out of 36 patients). On the other side of the tail of C-reactive protein distribution, if we consider the 18 patients with high inflammatory status, according to a C-reactive protein $>150 \mathrm{mg} / 1,11$ out of
18 patients (61\%) had DVT at LEDUS. These observations lead to speculate that the grade of systemic inflammation may be the key determinant facilitating DVT in COVID-19 and this is confirmed by the results of the multiple logistic regression analysis reported in Table 2 , showing that only C-reactive protein (not D-dimer or other clinical variables) is independently associated with DVT. According to our study, the finding of an extremely high or extremely low C-reactive protein value can certainly better inform the clinician reinforcing the decision to indicate LEDUS or not as a screening tool for DVT in a given COVID-19 patient.

C-reactive protein, but also age in this case, were independently significantly associated with the secondary end point of in-hospital mortality. In this regard, our study confirms the association between $\mathrm{C}$-reactive protein and mortality suggested at this time only in few pre-print reports (not yet published in peer-reviewed journals), in which age and inflammation severity were among the main key variables associated with mortality [19-22].

\section{Limitations}

The current study is a cross-sectional screening study, with the addition of the collection of short-term, in-hospital death follow-up, in which the available clinical and laboratory data were only the ones available from the chart or other electronic records. Cross-sectional studies cannot prove causation but only inform on associations detected.

The LEDUS was performed by voluntary trained radiologists operating at the bedside in the complex clinical contagious scenario of COVID-19 wards, so that the LEDUS images are not available for review, but only the written official report was stored, available from the electronic chart and radiology reporting system. Randomization of patients to be screened with LEDUS was directly performed by the radiologist in charge on the field, simply starting to scan patients either from the first room of the unit and on, or reverse from the last room, depending on the day, so that while this is theoretically not an ideal randomization process, it was the best that could be achieved during an infective outbreak and it should have avoided most types of selection bias. Several laboratory variables were available only in a percentage of the study population and consequently they could not be used in the current analysis, and among them, granular body mass index was not available in all patients, only the binary variable "obesity" found in the charts, defined by a body mass index $>29 \mathrm{~kg} / \mathrm{m}^{2}$ being available for all patients.

Funding This study was not supported by any funding. 


\section{Compliance with ethical standards}

Conflict of interest The authors declare that they have no conflict of interest.

Ethical standards All procedures performed in studies involving human participants were in accordance with the ethical standards of the institutional and/or national research committee and with the 1964 Helsinki Declaration and its later amendments or comparable ethical standards. The study was approved by our Institutional Review Board (Radcovid03/2020).

Informed consent The informed consent has been obtained from all patients.

\section{References}

1. Frizzelli A, Tuttolomondo D, Aiello M et al (2020) (2020) What happens to people's lungs when they get coronavirus disease 2019? Acta Biomed 91(2):146-149. https://doi.org/10.23750/ abm.v91i2.9574

2. Tuttolomondo D, Frizzelli A, Aiello M et al (2020) Beyond the lung involvement in COVID-19 patients. A review. Minerva Med. https://doi.org/10.23736/S0026-4806.20.06719-1 (Published online ahead of print, 2020 Jun 19)

3. Carotti M, Salaffi F, Sarzi-Puttini P et al (2020) Chest CT features of coronavirus disease 2019 (COVID-19) pneumonia: key points for radiologists. Radiol Med 125(7):636-646. https://doi. org/10.1007/s11547-020-01237-4

4. Gaia C, Maria Chiara C, Silvia L et al (2020) Chest CT for early detection and management of coronavirus disease (COVID-19): a report of 314 patients admitted to Emergency Department with suspected pneumonia. Radiol Med. https://doi.org/10.1007/s1154 7-020-01256-1 (Published online ahead of print, 2020 Jul 29)

5. Ierardi AM, Wood BJ, Arrichiello A et al (2020) Preparation of a radiology department in an Italian hospital dedicated to COVID19 patients. Radiol Med 125(9):894-901. https://doi.org/10.1007/ s11547-020-01248-1

6. Cozzi D, Albanesi M, Cavigli E et al (2020) Chest X-ray in new Coronavirus Disease 2019 (COVID-19) infection: findings and correlation with clinical outcome. Radiol Med 125(8):730-737. https://doi.org/10.1007/s11547-020-01232-9 (Epub 2020 Jun 9)

7. Helms J, Tacquard C, Severac F et al (2020) High risk of thrombosis in patients with severe SARS-CoV-2 infection: a multicenter prospective cohort study. Intensive Care Med. https://doi. org/10.1007/s00134-020-06062-x (Epub ahead of print)

8. Klok FA, Kruip MJHA, van der Meer NJM et al (2020a) Incidence of thrombotic complications in critically ill ICU patients with COVID-19. Thromb Res. https://doi.org/10.1016/j.throm res.2020.04.013 (Epub ahead of print)

9. Llitjos JF, Leclerc M, Chochois C et al (2020a) High incidence of venous thromboembolic events in anticoagulated severe COVID19 patients. J Thromb Haemost. https://doi.org/10.1111/jth.14869 (Epub ahead of print)

10. Mehta P, McAuley DF, Brown M et al (2020) COVID-19: consider cytokine storm syndromes and immunosuppression.
Lancet 395(10229):1033-1034. https://doi.org/10.1016/S0140 -6736(20)30628-0 (Epub 2020 Mar 16)

11. Borges AH, O’Connor JL, Phillips AN et al (2014) (2014) Factors associated with D-dimer levels in HIV-infected individuals. PLoS One. 9(3):e90978. https://doi.org/10.1371/journal.pone.0090978 (eCollection)

12. Ramacciotti E, Agati LB, Aguiar VCR et al (2019) Zika and chikungunya virus and risk for venous thromboembolism. Clin Appl Thromb Hemost. https://doi.org/10.1177/1076029618821184

13. Smither SJ, O'Brien LM, Eastaugh L et al (2019) Haemostatic changes in five patients infected with Ebola virus. Viruses. 11(7):E647. https://doi.org/10.3390/v11070647

14. Klok FA, Kruip MJHA, van der Meer NJM et al (2020b) Incidence of thrombotic complications in critically ill ICU patients with COVID-19. Thromb Res. https://doi.org/10.1016/j.throm res.2020.04.013 (Epub ahead of print)

15. Marone EM, Rinaldi LF (2020) Upsurge of deep vein thrombosis in patients affected by COVID-19: preliminary data and possible explanations. J Vasc Surg Venous Lymphat Disord. https://doi. org/10.1016/j.jvsv.2020.04.004 (Epub ahead of print)

16. Lodigiani C, Iapichino G, Carenzo L (2020) Venous and arterial thromboembolic complications in COVID-19 patients admitted to an academic hospital in Milan. Italy. Thromb Res. 191:9-14. https://doi.org/10.1016/j.thromres.2020.04.024 (Epub ahead of print)

17. Middeldorp S, Coppens M, van Haaps TF (2020) Incidence of venous thromboembolism in hospitalized patients with COVID19. J Thromb Haemost. https://doi.org/10.1111/jth.14888 (Epub ahead of print)

18. Llitjos JF, Leclerc M, Chochois C et al (2020b) High incidence of venous thromboembolic events in anticoagulated severe COVID19 patients. J Thromb Haemost. https://doi.org/10.1111/jth.14869 (Epub ahead of print)

19. Wynants L, Van Calster B, Bonten MMJ et al (2020) Prediction models for diagnosis and prognosis of covid-19 infection: systematic review and critical appraisal. BMJ 7(369):m1328. https://doi. org/10.1136/bmj.m1328

20. Lu J, Hu S, Fan R et al (2020) ACP risk grade: a simple mortality indexfor patients with confirmed or suspected severe acute respiratory syndrome coronavirus 2 disease (COVID-19) during the early stage of outbreak in Wuhan, China. medRxiv. https://doi. org/10.1101/2020.02.20.20025510 (Preprint)

21. Xie J, Hungerford D, Chen H et al (2020) Development and external validation of a prognostic multivariable model on admission for hospitalized patients with COVID-19. medRxiv. https://doi. org/10.1101/2020.03.28.20045997 (Preprint)

22. Yan L, Zhang H-T, Xiao Y et al (2020) Prediction of criticality in patients with severe Covid-19 infection using three clinical features: a machine learning-based prognostic model with clinical data in Wuhan. MedRxiv. https://doi. org/10.1101/2020.02.27.20028027 (Preprint)

Publisher's Note Springer Nature remains neutral with regard to jurisdictional claims in published maps and institutional affiliations. 